\title{
Hub-Induced Synchronization in Scale-Free Networks with Cluster Structure
}

\author{
Jianbao Zhang, ${ }^{1}$ Zhongjun $\mathrm{Ma}^{2}$ and Jinde $\mathrm{Cao}^{3,4}$ \\ ${ }^{1}$ School of Science, Hangzhou Dianzi University, Hangzhou 310018, China \\ ${ }^{2}$ School of Mathematics and Computing Science, Guilin University of Electronic Technology, Guilin 541004, China \\ ${ }^{3}$ Department of Mathematics, Southeast University, Nanjing 210096, China \\ ${ }^{4}$ Department of Mathematics, Faculty of Science, King Abdulaziz University, Jeddah 21589, Saudi Arabia \\ Correspondence should be addressed to Zhongjun Ma; mzj1234402@163.com
}

Received 18 January 2014; Revised 20 February 2014; Accepted 24 February 2014; Published 1 April 2014

Academic Editor: Hongli Dong

Copyright (c) 2014 Jianbao Zhang et al. This is an open access article distributed under the Creative Commons Attribution License, which permits unrestricted use, distribution, and reproduction in any medium, provided the original work is properly cited.

\begin{abstract}
A recent research indicated that the corticocortical connectivity network of the cat possesses cluster structure and that each cluster in the network is scale-free and has a most connected hub. Motivated by that research, we slightly modify the network model and derive sufficient conditions for cluster synchronization of the modified network based on Lyapunov function method. The obtained results indicate that cluster synchronization can be induced by the hubs of the scale-free networks. In our opinion, the concept of hubinduced synchronization provides a better understanding of cluster synchronization in scale-free networks. Numerical examples are provided to demonstrate the effectiveness of the theoretical results.
\end{abstract}

\section{Introduction}

In order to better understand the brain functions of mammals such as memory, language, and consciousness, many researches have been carried out to explore the organization and structure of the cerebral cortex [1-3]. These researches indicated that neurons in the surface of the cerebral cortex usually split into functional units (cortical areas) in the process of spreading information [1]. From a network point of view, these functional units form the nodes, linked by fibers, which form the network connections. For instance, there are 1139 corticocortical connections between 65 functional units in the cat cerebral cortex [2]. Recently, a network model was proposed to mimic the corticocortical connectivity of the cat and make clear the principles of organization and functioning of the cerebral cortex [3]. Based on statistical analysis, the network model is shown to exhibit a cluster structure with each cluster being scale-free, neither fully regular, nor completely random $[4,5]$. Each of these clusters composed by cortical areas forms an organization playing common functional roles. Previous evidences indicated that each cluster possesses a cluster hub with the following major features: (1) each cluster possessing only one hub, (2) every hub possessing a large density of connections, and (3) all hubs being connected among themselves in a strong fashion $[3,6]$. Therefore, the network model is called a rich-club clustered network. Under the assumption that all the hubs are all-to-all coupled, it was shown that the scale-free structure of the network contributes a great deal to suppress bursting synchronization [3].

Not coincidentally, it has been demonstrated that many real world large-scale networks display a scale-free feature, such as biological networks, World Wide Webs, and interbank payment networks. Generally speaking, the scale-free feature of real networks is believed to stem from preferential attachment growth $[4,5]$. In the event, the degree distribution of the networks obeys a power-law form: a large number of nodes are connected sparsely, while a small number of nodes (hubs) are connected densely. In the past decades, synchronization in coupled oscillator networks has attracted much attention $[7,8]$. Obviously, these hubs will play a key role in the process of synchronization. It has been shown that the synchronizability of a scale-free network is fragile to specific removal of hubs and robust against the removal of nonhub nodes [9]. Recently, in scale-free networks composed of Kuramoto oscillators, explosive synchronization has been 
studied based on mean-field approximations [10, 11]. A prerequisite for the researches is that the natural frequency of each oscillator should be equal to its node degree, which implies that the natural frequencies of the hubs should be higher than the other oscillators $[10,11]$. All in all, for scalefree networks, there is an intimate relationship between synchronization and the couplings or dynamics of the hubs.

For a general network, cluster structure implies that the oscillators are divided into clusters, and the connections within each cluster are dense, while the connections between different clusters are sparse [12-14]. As the result, cluster synchronization usually occurs in networks with cluster structure; that is, synchronization occurs in each cluster but may not occur among different clusters [15-21]. In order to study cluster synchronization, two control methods have been proposed. The first one is the pinning control strategy [2224], which has been applied to realize an expected cluster synchronization pattern in networks with cluster structure [15] and in neural networks with stochastic delay [16]. Another method is to impose suitable constraints on the coupling matrix $[17,18]$. As special cases, it has also been shown that cluster synchronization can be achieved by constructing nontrivial coupling matrices [19-21]. To the best of our knowledge, few researches have focused on cluster synchronization in scale-free networks. However, many researches indicated that some of scale-free networks possess cluster structure $[25,26]$. Therefore, it is necessary to explore cluster synchronization in scale-free networks, especially to study the relation between the couplings of cluster hubs and cluster synchronization.

In this paper, we aim to model the cat cortex structure through building a coupled oscillator network with a scalefree feature and to study cluster synchronization in such a network. In contrast with the statistical analysis in previous researches, this paper carries out rigorous theoretical investigations on collective dynamics of the cerebral cortex of cats or other mammals. At first, we build a network model with cluster structure, in which each cluster has a most connected cluster hub. The obtained network model fully reflects the characteristics of the cerebral cortex of cats. Secondly, we derive the error systems between each node and its cluster hub. Based on stability analysis of the error systems, we obtain the criterion for cluster synchronization of the network. Motivated by the recent researches on synchronization in scale-free networks [9-11], we focus on analyzing the key role of the cluster hubs in process of cluster synchronization. The obtained criterion shows that the couplings from the cluster hubs are of great significance for realizing cluster synchronization. In other words, cluster synchronization can be induced by the cluster hubs. Therefore, we call this type of synchronization as hub-induced synchronization, which provides a better understanding of cluster synchronization in scale-free networks.

The rest of this paper is organized as follows. In Section 2, the model of a clustered scale-free network with cluster hubs is introduced, and some preliminaries are introduced. In Section 3, the main result on hub-induced synchronization in the network model is obtained. In Section 4, numerical examples are given to show the effectiveness of the theoretical results. Finally, the paper is concluded by a brief discussion in Section 5.

\section{Preliminaries}

2.1. A Scale-Free Network with Cluster Hubs. Consider the following network with cluster structure:

$$
\dot{x}_{i}=f\left(x_{i}, t\right)+\varepsilon \sum_{l=1}^{d} \sum_{j \in P_{l}} c_{i j} H x_{j},
$$

where $x_{i}=\left(x_{i}^{1}, \ldots, x_{i}^{n}\right)^{\top}$ is the coordinate of the $i$ th oscillator, $f: R^{n} \times[0,+\infty) \rightarrow R^{n}$ is a continuous map, $\varepsilon>0$ is the coupling strength, $H=\operatorname{diag}\left(h_{1}, \ldots, h_{n}\right)$ is a nonnegative matrix, and $C=\left(c_{i j}\right)_{m \times m}$ is the coupling matrix satisfying $\sum_{j=1}^{m} c_{i j}=0, i=1, \ldots, m$. Without loss of generality, suppose that $P_{1}=\left\{1, \ldots, \sigma_{1}\right\}, \ldots, P_{d}=\left\{\sigma_{d-1}+1, \ldots, \sigma_{d}\right\}$, and $P=$ $\left\{P_{1}, \ldots, P_{d}\right\}$. Then the coupling matrix $C$ is partitioned into blocks:

$$
C=\left[\begin{array}{ccc}
C_{11} & \cdots & C_{1 d} \\
\cdots & \cdots & \cdots \\
C_{d 1} & \cdots & C_{d d}
\end{array}\right],
$$

where $C_{k l} \in R^{p_{k} \times p_{l}}, p_{k}=\sigma_{k}-\sigma_{k-1}, p_{0}=0$. Assume that each cluster has a single most connected node, which is called a cluster hub. That is to say, the following assumption holds.

$\left(H_{1}\right)$ Each cluster $P_{k}$ has a cluster hub $\sigma_{k}$, and there holds

$$
c_{\sigma_{\hat{i}} j}+c_{\sigma_{\hat{j}} i} \leq c_{i j}+c_{j i} \text {, }
$$

where $\hat{i}=k$ if $i \in P_{k}, i, j=1, \ldots, m, \hat{i} \neq \hat{j}$.

The assumption $\left(H_{1}\right)$ means that, for any pair of nodes $i, j$ belonging to different clusters, the sum of the coupling from $j$ to $\sigma_{\hat{i}}$ (the cluster hub of $i$ ) and the coupling from $i$ to $\sigma_{\hat{j}}$ (the cluster hub of $j$ ) should be less than the sum of the couplings between $i$ and $j$. That is to say, each cluster hub should not be influenced more greatly by the nodes in other clusters. Otherwise, it should be regarded as the cluster hub of these nodes. In order to understand the assumptions more clearly, we can refer to Figure 1 in Section 4. As can be seen from Figure 1, each cluster hub is coupled densely with the nodes belonging to its cluster and coupled sparsely with the nodes belonging to other clusters. Therefore, it is natural to propose the assumption $\left(H_{1}\right)$. In a particular case, when each cluster hub is only coupled with the nodes belonging to its cluster, not coupled with the nodes belonging to other clusters, the assumption $\left(H_{1}\right)$ is satisfied because the left hand of the inequality is equal to zero.

2.2. The Cluster Synchronization Manifold. Now, we introduce the concept of the cluster synchronization manifold and its attractiveness.

Definition 1. The set

$$
\mathbb{M}(P)=\left\{\left(x_{1}^{\top}, \ldots, x_{m}^{\top}\right)^{\top}: x_{i}=x_{j} \in R^{n} \text { if } \hat{i}=\hat{j}\right\}
$$

is called the cluster synchronization manifold corresponding to the partition $P$. 
Definition 2. The cluster synchronization manifold $\mathbb{M}(P)$ is globally attractive for the system (1), or cluster synchronization corresponding to the partition $P$ occurs, if

$$
\lim _{t \rightarrow+\infty} \sum_{k=1}^{d} \sum_{i \in P_{k}}\left\|x_{i}-x_{\sigma_{k}}\right\|=0
$$

holds for any initial condition $\left(x_{1}^{\top}(0), \ldots, x_{m}^{\top}(0)\right)^{\top}$, where $\|\cdot\|$ denotes the 2-norm of vectors.

Before studying the attractiveness of the manifold $\mathbb{M}(P)$, a sufficient and necessary condition for its invariance is introduced as follows.

Lemma 3 (see [17]). The cluster synchronization manifold $\mathbb{M}(P)$ is an invariant manifold of the system (1) if and only if every submatrix $C_{k l}$ in the form (2) has equal-row-sums, $k, l=1, \ldots, d$.

According to Lemma 3, the following assumption [17, 18] is supposed to hold throughout this paper.

$\left(H_{2}\right)$ Every submatrix $C_{k l} \in R^{p_{k} \times p_{l}}$ in the form (2) has equal-row-sums, $k, l=1, \ldots, d$.

In the next section, we will derive sufficient conditions for cluster synchronization of the system (1) induced by the cluster hubs based on the preliminaries mentioned above.

\section{Cluster Synchronization Induced by Cluster Hubs}

Defining the synchronization errors as $X_{\sigma_{\tilde{i}} i}=x_{i}-x_{\sigma_{\tilde{i}}}, i=$ $1, \ldots, m$, we get the error system

$$
\begin{aligned}
& \dot{X}_{\sigma_{\hat{i}} i}=\dot{x}_{i}-\dot{x}_{\sigma_{\hat{i}}} \\
& =f\left(x_{i}, t\right)+\varepsilon \sum_{l=1}^{d} \sum_{j \in P_{l}} c_{i j} H x_{j}-f\left(x_{\sigma_{\hat{i}}}, t\right)-\varepsilon \sum_{l=1}^{d} \sum_{j \in P_{l}} c_{\sigma_{\hat{i}} j} H x_{j} \\
& =f\left(x_{i}, t\right)-f\left(x_{\sigma_{\widehat{i}}}, t\right)+\varepsilon \sum_{l=1}^{d} \sum_{j \in P_{l}} c_{i j} H X_{\sigma_{\widehat{j}} j} \\
& -\varepsilon \sum_{l=1}^{d} \sum_{j \in P_{l}} c_{\sigma_{\hat{i}} j} H X_{\sigma_{\hat{j}} j}+\varepsilon \sum_{l=1}^{d} \sum_{j \in P_{l}} c_{i j} H x_{\sigma_{\widehat{j}}}-\varepsilon \sum_{l=1}^{d} \sum_{j \in P_{l}} c_{\sigma_{\hat{i}} j} H x_{\sigma_{\hat{j}}} \\
& =f\left(x_{i}, t\right)-f\left(x_{\sigma_{\widehat{i}}}, t\right)+\varepsilon \sum_{l=1}^{d} \sum_{j \in P_{l}}\left(c_{i j}-c_{\sigma_{\widehat{i}} j}\right) H X_{\sigma_{\widehat{j}} j} \\
& +\varepsilon \sum_{l=1}^{d}\left[\sum_{j \in P_{l}}\left(c_{i j}-c_{\sigma_{\hat{i}} j}\right)\right] H x_{\sigma_{l}} .
\end{aligned}
$$

It follows from the assumption $\left(\mathrm{H}_{2}\right)$ that

$$
\dot{X}_{\sigma_{\tilde{i}} i}=f\left(x_{i}, t\right)-f\left(x_{\sigma_{\widehat{i}}}, t\right)+\varepsilon \sum_{l=1}^{d} \sum_{j \in P_{l}} a_{i j} H X_{\sigma_{\tilde{j}} j},
$$

where $a_{i j}=c_{i j}-c_{\sigma_{\tilde{i}}}$. Decompose the matrix $A=\left(a_{i j}\right)$ as follows:

$$
A=A_{\alpha}+A_{\beta}+A_{\gamma}+A_{\xi}
$$

where

$$
\begin{aligned}
& A_{\alpha}=\left\{\alpha_{i j}\right\}: \begin{cases}0, & \hat{i} \neq \hat{j} ; \\
\alpha_{i j}=\frac{\left(a_{i j}+a_{j i}\right)}{2}, & \hat{i}=\widehat{j}, i \neq j ; \\
\alpha_{i j}=-\sum_{q=1, q \neq i}^{m} \alpha_{i q}, & i=j\end{cases} \\
& A_{\beta}=\left\{\beta_{i j}\right\}: \begin{cases}\beta_{i j}=\frac{\left(a_{i j}+a_{j i}\right)}{2}, & \widehat{i} \neq \hat{j} ; \\
0, & \hat{i}=\widehat{j}, i \neq j ; \\
\beta_{i j}=-\sum_{q=1, q \neq i}^{m} \beta_{i q}, & i=j,\end{cases}
\end{aligned}
$$

are symmetric and zero-row-sum matrices, and

$$
A_{\gamma}=\left\{\gamma_{i j}\right\}: \begin{cases}\gamma_{i j}=\frac{\left(a_{i j}-a_{j i}\right)}{2}, & i \neq j ; \\ \gamma_{i j}=0, & i=j\end{cases}
$$

is an antisymmetric matrix, and $A_{\xi}=\operatorname{diag}\left\{\xi_{11}, \ldots, \xi_{m m}\right\}$, where $\xi_{i i}=a_{i i}-\alpha_{i i}-\beta_{i i}, i=1, \ldots, m$.

Noticing that $X_{\sigma_{\tilde{i}} \sigma_{\hat{i}}}=0$, we introduce the matrices

$$
\bar{A}_{k}=\left[\begin{array}{ccc}
\alpha_{\sigma_{k-1}+1, \sigma_{k-1}+1} & \cdots & \alpha_{\sigma_{k-1}+1, \sigma_{k}-1} \\
\cdots & \cdots & \cdots \\
\alpha_{\sigma_{k}-1, \sigma_{k-1}+1} & \cdots & \alpha_{\sigma_{k}-1, \sigma_{k}-1}
\end{array}\right]=\left\{\alpha_{i j}\right\}_{i, j \in \bar{P}_{k}},
$$

where $\bar{P}_{k}=\left\{\sigma_{k-1}+1, \ldots, \sigma_{k}-1\right\}, k=1, \ldots, d$. For the convenience of later use, we denote

$$
\begin{gathered}
X=\left(X_{\sigma_{1} 1}^{\top}, \ldots, X_{\sigma_{\bar{m}} m}^{\top}\right)^{\top} \in R^{m n}, \\
\bar{X}_{k}^{s}=\left(X_{\sigma_{k}, \sigma_{k-1}+1}^{s}, \ldots, X_{\sigma_{k}, \sigma_{k}-1}^{s}\right)^{\top} \in R^{n\left(p_{k}-1\right)},
\end{gathered}
$$

where $s=1, \ldots, n, k=1, \ldots, d$. Then based on Lyapunov function method, we obtain the following theorem and the corresponding proof.

Theorem 4. Let $Q=\operatorname{diag}\left(q_{1}, \ldots, q_{n}\right)$ be a positive-definite diagonal matrix, let $\Delta=\operatorname{diag}\left(\delta_{1}, \ldots, \delta_{n}\right)$ be a diagonal matrix with $\delta_{j} \leq 0$ for $j \in\left\{j: h_{j}=0\right\}$, and the function $f(u, t)$ satisfies

$$
\begin{gathered}
(u-v)^{\top} Q[f(u, t)-f(v, t)-\Delta(u-v)] \\
\leq-\epsilon(u-v)^{\top}(u-v),
\end{gathered}
$$

for some $\epsilon>0$, all $u, v \in R^{n}$, and all $t \geq 0$. Then under the assumptions $\left(H_{1}\right)$ and $\left(H_{2}\right)$, cluster synchronization occurs in the system (1) if

$$
\delta_{s}+\varepsilon \max _{i \in \bar{P}_{k}}\left\{a_{i i}-\alpha_{i i}-\beta_{i i}\right\} h_{s}+\varepsilon h_{s} \max \lambda\left(\bar{A}_{k}\right) \leq 0,
$$


where $\lambda_{\max }\left(\bar{A}_{k}\right)$ is the largest eigenvalue of $\bar{A}_{k}, k=1, \ldots, d$, $s=1, \ldots, n$.

Proof. Considering the Lyapunov function,

$$
V(X)=\frac{1}{2} \sum_{k=1}^{d} \sum_{i \in P_{k}} X_{\sigma_{\hat{i}} i}^{\top} Q X_{\sigma_{\hat{i}}},
$$

we have

$$
\begin{aligned}
\dot{V}(X)= & \sum_{k=1}^{d} \sum_{i \in P_{k}} X_{\sigma_{\hat{i}} i}^{\top} Q \\
& \times\left[f\left(x_{i}, t\right)-f\left(x_{\hat{i}}, t\right)+\varepsilon \sum_{l=1}^{d} \sum_{j \in P_{l}} a_{i j} H X_{\sigma_{\hat{j}} j}\right] \\
= & \sum_{k=1}^{d} \sum_{i \in P_{k}} X_{\sigma_{\hat{i}} i}^{\top} Q\left[f\left(x_{i}, t\right)-f\left(x_{\hat{i}}, t\right)-\Delta X_{\sigma_{\hat{i}} i}\right. \\
\leq & -\epsilon \sum_{k=1}^{d} \sum_{i \in P_{k}} X_{\sigma_{\hat{i}} i}^{\top} X_{\sigma_{\hat{i}} i} \\
& +\sum_{k=1}^{d} \sum_{i \in P_{k}} X_{\sigma_{\hat{i}} i}^{\top} Q\left(\Delta X_{\sigma_{\hat{i}^{i}} i}+\varepsilon \sum_{l=1}^{d} \sum_{j \in P_{l}} a_{i j} H X_{\sigma_{\hat{j}} j}\right] \\
\leq & -\frac{2 \epsilon V(X)}{\left.\max _{1 \leq i \leq n} \sum_{l=1} \sum_{j \in P_{l}} a_{i j} H X_{\sigma_{\hat{j}} j}\right)} \\
& +S .
\end{aligned}
$$

By the decomposition (8), it is clear that

$$
\begin{aligned}
S= & \sum_{k=1}^{d} \sum_{i \in P_{k}} X_{\sigma_{\hat{i}} i}^{\top} Q \Delta X_{\sigma_{\hat{i}} i} \\
& +\varepsilon \sum_{k=1}^{d} \sum_{l=1}^{d} \sum_{i \in P_{k}} \sum_{j \in P_{l}}\left(\alpha_{i j}+\beta_{i j}+\gamma_{i j}+\xi_{i j}\right) X_{\sigma_{\hat{i}} i}^{\top} Q H X_{\sigma_{\hat{j}} j} \\
= & S_{\delta}+S_{\alpha}+S_{\beta}+S_{\gamma}+S_{\xi} .
\end{aligned}
$$

Firstly, noticing the antisymmetry of the matrix $A_{\gamma}$, we have

$$
\begin{aligned}
S_{\gamma}= & \varepsilon \sum_{k=1}^{d} \sum_{l=1}^{d} \sum_{i \in P_{k}} \sum_{j \in P_{l}} \gamma_{i j} X_{\sigma_{i} i}^{\top} Q H X_{\sigma_{\hat{j}} j} \\
= & \varepsilon \sum_{k=1}^{d-1} \sum_{l=k+1}^{d} \sum_{i \in P_{k}} \sum_{j \in P_{l}} \gamma_{i j} X_{\sigma_{\hat{i}}}^{\top} Q H X_{\sigma_{\hat{j}} j} \\
& +\varepsilon \sum_{l=1}^{d-1} \sum_{k=l+1}^{d} \sum_{i \in P_{k}} \sum_{j \in P_{l}} \gamma_{i j} X_{\sigma_{i}}^{\top} Q H X_{\sigma_{\hat{j}} j}
\end{aligned}
$$

$$
\begin{aligned}
= & \varepsilon \sum_{k=1}^{d-1} \sum_{l=k+1}^{d} \sum_{i \in P_{k}} \sum_{j \in P_{l}} \gamma_{i j} X_{\sigma_{\hat{i}} i}^{\top} Q H X_{\sigma_{\hat{j}} j} \\
& +\varepsilon \sum_{k=1}^{d-1} \sum_{l=k+1}^{d} \sum_{j \in P_{l}} \sum_{i \in P_{k}} \gamma_{j i} X_{\sigma_{\hat{j}} j}^{\top} Q H X_{\sigma_{\hat{i}} i} \\
= & \varepsilon \sum_{k=1}^{d-1} \sum_{l=k+1}^{d} \sum_{i \in P_{k}} \sum_{j \in P_{l}}\left(\gamma_{i j}+\gamma_{j i}\right) X_{\sigma_{\hat{j} j} j}^{\top} Q H X_{\sigma_{\hat{i}} i}=0 .
\end{aligned}
$$

Secondly, noticing the zero-row-sum of the matrix $A_{\beta}$, we have

$$
\begin{aligned}
& S_{\beta}=\varepsilon \sum_{k=1}^{d} \sum_{l=1}^{d} \sum_{i \in P_{k}} \sum_{j \in P_{l}} \beta_{i j} X_{\sigma_{\bar{i}} i}^{\top} Q H\left(X_{\sigma_{\hat{j}} j}-X_{\sigma_{\hat{i}}}\right) \\
& =\varepsilon \sum_{k=1}^{d-1} \sum_{l=k+1}^{d} \sum_{i \in P_{k}} \sum_{j \in P_{l}} \beta_{i j} X_{\sigma_{\hat{i}}}^{\top} Q H\left(X_{\sigma_{\hat{j}} j}-X_{\sigma_{\hat{i}}}\right) \\
& +\varepsilon \sum_{l=1}^{d-1} \sum_{k=l+1}^{d} \sum_{i \in P_{k}} \sum_{j \in P_{l}} \beta_{i j} X_{\sigma_{\hat{i}}}^{\top} Q H\left(X_{\sigma_{\hat{j}} j}-X_{\sigma_{\hat{i}} i}\right) \\
& =\varepsilon \sum_{k=1}^{d-1} \sum_{l=k+1}^{d} \sum_{i \in P_{k}} \sum_{j \in P_{l}} \beta_{i j} X_{\sigma_{\hat{i}}}^{\top} Q H\left(X_{\sigma_{\hat{j}} j}-X_{\sigma_{\hat{i}}^{i}}\right) \\
& +\varepsilon \sum_{k=1}^{d-1} \sum_{l=k+1}^{d} \sum_{j \in P_{l}} \sum_{i \in P_{k}} \beta_{j i} X_{\sigma_{\hat{j} j} j}^{\top} Q H\left(X_{\sigma_{\hat{i}} i}-X_{\sigma_{\hat{j}} j}\right) \\
& =-\varepsilon \sum_{k=1}^{d-1} \sum_{l=k+1}^{d} \sum_{i \in P_{k}} \sum_{j \in P_{l}} \beta_{i j}\left(X_{\sigma_{\hat{j}} j}-X_{\sigma_{\hat{i}}}\right)^{\top}
\end{aligned}
$$

$$
\times Q H\left(X_{\sigma_{\tilde{j} j}}-X_{\sigma_{\hat{i}}}\right) .
$$

Based on the assumption $\left(H_{1}\right)$, we obtain $S_{\beta} \leq 0$. Therefore,

$$
\begin{aligned}
& S \leq S_{\delta}+S_{\alpha}+S_{\xi} \\
&=\sum_{k=1}^{d} \sum_{i \in P_{k}} X_{\sigma_{\hat{i}}^{i}}^{\top} Q\left[\Delta X_{\sigma_{\hat{i}} i}+\varepsilon \sum_{j \in P_{k}} \alpha_{i j} H X_{\sigma_{\hat{j}} j}\right. \\
&\left.+\varepsilon\left(a_{i i}-\alpha_{i i}-\beta_{i i}\right) H X_{\sigma_{\hat{i}} i}\right] \\
& \leq \sum_{k=1}^{d} \sum_{i \in \bar{P}_{k}} X_{\sigma_{\hat{i}}}^{\top} Q[ {\left[\left(\Delta+\varepsilon \max _{i \in \bar{P}_{k}}\left\{a_{i i}-\alpha_{i i}-\beta_{i i}\right\} H\right) X_{\sigma_{\hat{i}} i}\right.} \\
&\left.+\varepsilon \sum_{j \in \bar{P}_{k}} \alpha_{i j} H X_{\sigma_{\hat{j} j}}\right]
\end{aligned}
$$




$$
\begin{aligned}
=\sum_{k=1}^{d} \sum_{s=1}^{n} q_{s} \bar{X}_{k}^{s \top}\left[\left(\delta_{s}+\varepsilon \max _{i \in \bar{P}_{k}}\left\{a_{i i}-\alpha_{i i}-\beta_{i i}\right\} h_{s}\right) I_{p_{k}-1}\right. \\
\left.+\varepsilon h_{s} \bar{A}_{k}\right] \bar{X}_{k}^{s} .
\end{aligned}
$$

It is well known that a symmetric matrix $\bar{A}_{k}$ has the decomposition $\bar{A}_{k}=U_{k} \Lambda_{k} U_{k}^{\top}$, where $U_{k} \in R^{\left(p_{k}-1\right) \times\left(p_{k}-1\right)}$ is a unitary matrix and $\Lambda_{k}=\operatorname{diag}\left\{\lambda_{1}\left(\bar{A}_{k}\right), \ldots, \lambda_{p_{k}-1}\left(\bar{A}_{k}\right)\right\}$ is a real diagonal matrix. Applying the orthogonal transformations

$$
\bar{X}_{k}^{s}=U_{k} Y_{k}^{s}, \quad k=1, \ldots, d, s=1, \ldots, n,
$$

to $(20)$, we finally get

$$
\begin{aligned}
S \leq \sum_{k=1}^{d} \sum_{s=1}^{n} q_{s} Y_{k}^{s \top}[ & \left(\delta_{s}+\varepsilon \max _{i \in \bar{P}_{k}}\left\{a_{i i}-\alpha_{i i}-\beta_{i i}\right\} h_{s}\right) \\
& \left.\times I_{p_{k}-1}+\varepsilon h_{s} \Lambda_{k}\right] Y_{k}^{s} .
\end{aligned}
$$

Therefore, the conditions in Theorem 4 are sufficient for $S \leq$ 0 , and then

$$
\dot{V}(X) \leq \frac{-2 \epsilon V(X)}{\max _{1 \leq i \leq n} q_{i}} .
$$

This completes the proof of Theorem 4 .

In order to better understand the condition (14), we make the following analysis:

$$
\begin{aligned}
a_{i i}- & \alpha_{i i}-\beta_{i i} \\
& =c_{i i}-c_{\hat{i i}}+\frac{1}{2} \sum_{j=1, j \neq i}^{m}\left(a_{i j}+a_{j i}\right) \\
& =-\sum_{j=1, j \neq i}^{m} c_{i j}-c_{\hat{i i}}+\frac{1}{2} \sum_{j=1, j \neq i}^{m}\left(c_{i j}+c_{j i}-c_{\hat{i} j}-c_{\hat{j i}}\right) \\
& =\frac{1}{2} \sum_{j=1, j \neq i}^{m}\left(c_{j i}-c_{i j}\right)-\frac{1}{2} \sum_{j=1, j \neq i}^{m}\left(c_{\hat{i} j}+c_{\hat{j i}}\right)-c_{i i} .
\end{aligned}
$$

Combining the definitions of the matrices $\bar{A}_{k}$ in (11), we can conclude that cluster synchronization can be realized by increasing the couplings of the cluster hubs. Therefore, this type of cluster synchronization is called as hub-induced synchronization.

\section{Numerical Simulations}

Consider the system (1) composed of $m$ neural networks:

$$
\dot{x}_{i}=-D x_{i}+T g\left(x_{i}\right)+\varepsilon \sum_{j=1}^{m} c_{i j} H x_{j}, \quad i=1, \ldots, m,
$$

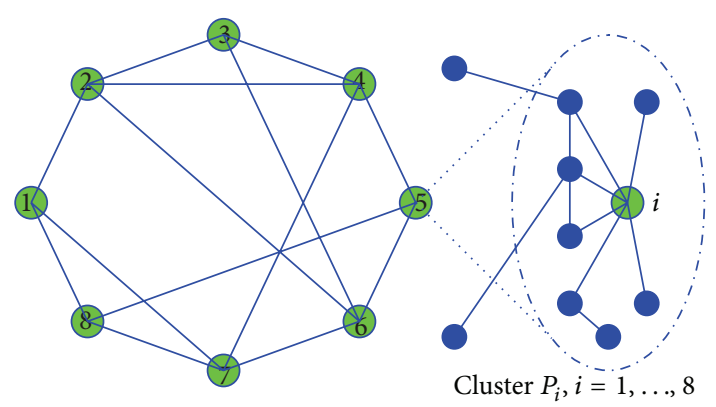

FIGURE 1: Schematic diagram of a network model possessing scalefree feature and cluster structure. Each cluster has a most connected hub.

where $x_{i} \in R^{3}, D=H=I_{3}, g\left(x_{i}\right)=\left(g\left(x_{i}^{1}\right), g\left(x_{i}^{2}\right), g\left(x_{i}^{3}\right)\right)^{\top}$, $g(s)=(|s+1|-|s-1|) / 2$, and

$$
T=\left(\begin{array}{ccc}
1.25 & -3.2 & -3.2 \\
-3.2 & 1.1 & -4.4 \\
-3.2 & 4.4 & 1.0
\end{array}\right)
$$

By using Matlab LMI Control Toolbox, one can obtain that $\delta_{\min }=5.685$ satisfies the condition (13) [27].

4.1. Simulation 1. Motivated by the "rich-club" clustered network in [3], we consider a network model possessing scale-free feature, cluster structure, in which each cluster has a most connected hub. For example, a schematic diagram of such a network consisting of 64 nodes is shown in Figure 1. These nodes split into 8 clusters, and each cluster displays a scale-free feature.

Define the synchronization errors

$$
e_{0}=\sum_{j=1}^{64} \frac{\left|x_{j}-x_{1}\right|}{64}, \quad e_{i}=\sum_{j \in P_{i}}\left|x_{j}-x_{j}\right|, \quad i=1,2, \ldots, 8,
$$

where the time evolutions are plotted in Figure 2. As can be seen, the system (25) realizes cluster synchronization. Obviously, the hubs of the system (25) play key roles in realizing cluster synchronization.

4.2. Simulation 2. It is worth noting that Theorem 4 is also valid even for a general network without a scale-free feature. If the oscillators in a network split into several clusters and each cluster has a most connected cluster hub, cluster synchronization can be realized by controlling the cluster hubs. For example, consider the coupling matrix:

$$
C=\left(\begin{array}{ccccc}
-c & -1 & c & 1 & 0 \\
-1 & -c & c & 0 & 1 \\
0 & 0 & -1 & 1 & 0 \\
-1 & 0 & 1 & -c & c \\
0 & -1 & 1 & 0 & 0
\end{array}\right)
$$

It is not difficult to verify that the matrix $C$ and the partition $P=\{1,2,3,4,5\}$ satisfy the assumptions $\left(H_{1}\right)$ and $\left(H_{2}\right)$. 


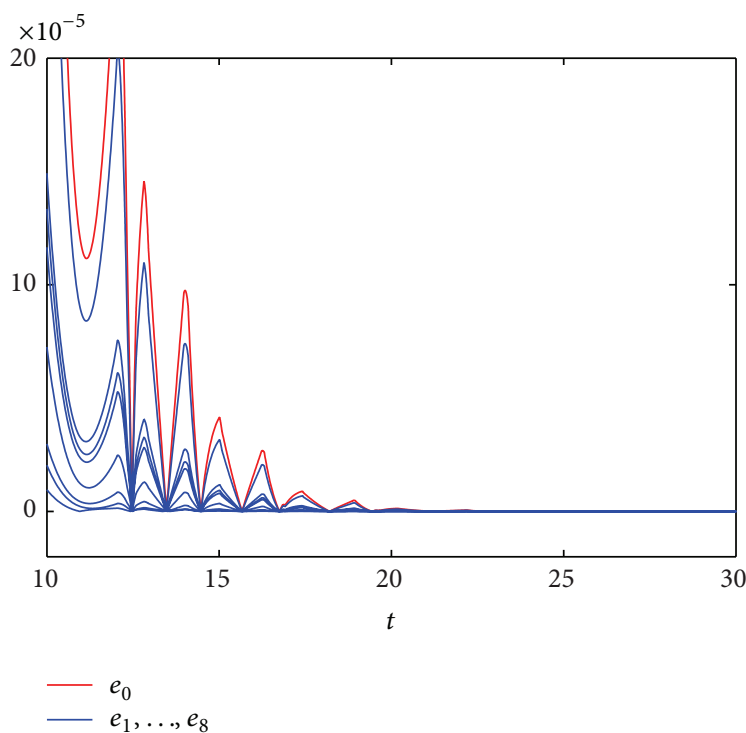

FIgURE 2: Time evolution of the errors $e_{0}$ and $e_{i}, i=1,2, \ldots, 8$.

The third node is the cluster hub of $P_{1}$, and the fifth node is the cluster hub of $P_{2}$. And the matrices corresponding to (11) are

$$
\bar{A}_{1}=\left(\begin{array}{ll}
-c & -1 \\
-1 & -c
\end{array}\right), \quad \bar{A}_{2}=(-c) .
$$

Then we can conclude from Theorem 4 that the threshold for cluster synchronization of the system (25) with the coupling matrix (28) is $\varepsilon \geq \delta_{\min } /(c-1)$.

By taking $\varepsilon=\delta_{\min } / 24, c=25$, and defining

$$
\begin{gathered}
e_{0}=\sum_{i=1}^{5} \frac{\left|x_{i}-x_{1}\right|}{5}, \\
e_{1}=\sum_{i=2}^{3}\left|x_{i}-x_{1}\right|, \quad e_{2}=\left|x_{5}-x_{4}\right|,
\end{gathered}
$$

the time evolution of the synchronization errors is plotted in Figure 3. In order to study synchronization induced by the hubs, we fix $\varepsilon=0.04$ and increase the parameter $c$ from 5 to 100 gradually. Figure 4 is plotted to show the variations of the synchronization errors. As can be seen, the errors $e_{1}$ and $e_{2}$ tend to zero when $c \geq 20$, which implies that cluster synchronization occurs. Therefore, the validity of Theorem 4 is confirmed.

\section{Conclusion}

In order to mimic the topology structure of the corticocortical connectivity network, this paper has proposed a network model with a scale-free feature, cluster structure, and the property that each cluster has a cluster hub. Then we decompose the coupling matrix into four matrices and derive an error system of the network. By a detailed stability analysis of the error system, we have obtained sufficient conditions to achieve cluster synchronization. The obtained result indicates

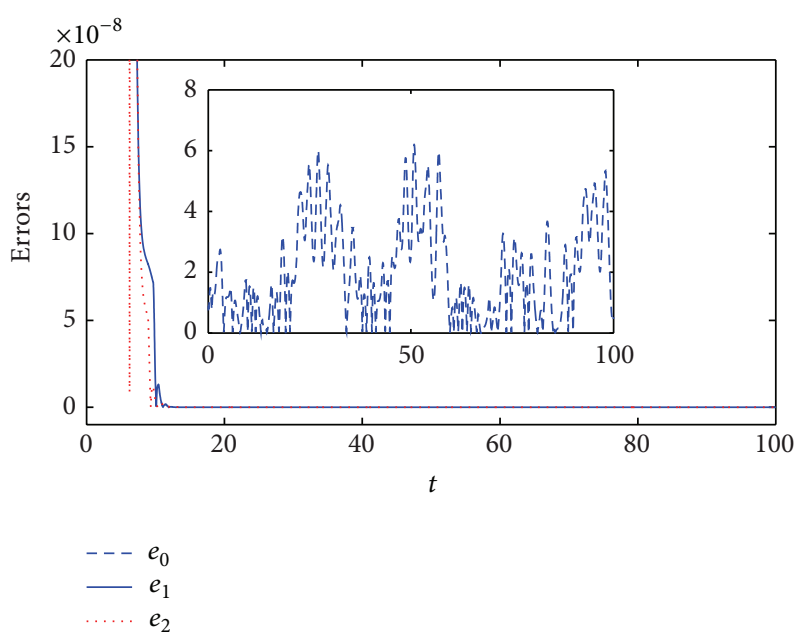

Figure 3: Time evolution of the errors $e_{0}, e_{1}$, and $e_{2}$.

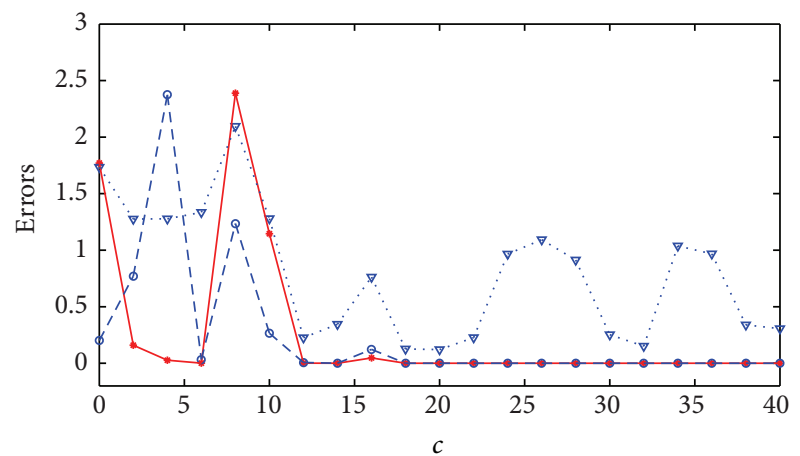

$$
\begin{aligned}
& * e_{1} \\
& \bigcirc e_{2} \\
& \nabla e_{0}
\end{aligned}
$$

FIGURE 4: Dependence of the synchronization errors on $c \in[0,40]$, where $\varepsilon=0.04$.

that cluster synchronization can be guaranteed by increasing the coupling strength of the cluster hubs.

Many previous researches have shown that bursting synchronization can also be suppressed by imposing an external controlling intervention $[3,6]$. It may be an interesting challenge to discuss cluster synchronization of the modified "richclub" network (1) with an external controlling intervention applied to cluster hubs. The controlling intervention could be a time-delayed feedback signal, a constantly applied pulse, or an alternating on-off pulse. Further exploration into this topic will help us better understand the relation between the couplings of cluster hubs and cluster synchronization.

\section{Conflict of Interests}

The authors declare that they have no conflict of interests regarding the publication of this paper. 


\section{Acknowledgments}

This project is supported by the NNSF of China (nos. 11162004,61203155 and 11171084), the NSF of Zhejiang Province (no. LQ12A01003 and LQ12A01002), and the NSF of Guangxi Province (no. 2013GXNSFAA019006).

\section{References}

[1] P. Rakic, "Specification of cerebral cortical areas," Science, vol. 241, no. 4862, pp. 170-176, 1988.

[2] J. W. Scannell, C. Blakemore, and M. P. Young, "Analysis of connectivity in the cat cerebral cortex," Journal of Neuroscience, vol. 15, no. 2, pp. 1463-1483, 1995.

[3] E. L. Lameu, C. A. S. Batista, A. M. Batista et al., "Suppression of bursting synchronization in clustered scale-free (rich-club) neuronal networks," Chaos, vol. 22, no. 4, Article ID 043149, 12 pages, 2012.

[4] R. Albert and A. L. Barabási, "Statistical mechanics of complex networks," Reviews of Modern Physics, vol. 74, no. 1, pp. 47-97, 2002.

[5] M. E. J. Newman, "The structure and function of complex networks," SIAM Review, vol. 45, no. 2, pp. 167-256, 2003.

[6] C. A. S. Batista, S. R. Lopes, R. L. Viana, and A. M. Batista, "Delayed feedback control of bursting synchronization in a scale-free neuronal network," Neural Networks, vol. 23, no. 1, pp. 114-124, 2010.

[7] J. Lu, D. W. C. Ho, J. Cao, and J. Kurths, "Exponential synchronization of linearly coupled neural networks with impulsive disturbances," IEEE Transactions on Neural Networks, vol. 22, no. 2, pp. 329-336, 2011.

[8] J. Lu, D. W. C. Ho, and J. Cao, "A unified synchronization criterion for impulsive dynamical networks," Automatica, vol. 46, no. 7, pp. 1215-1221, 2010.

[9] X. F. Wang and G. R. Chen, "Synchronization in scale-free dynamical networks: robustness and fragility," IEEE Transactions on Circuits and Systems. I, vol. 49, no. 1, pp. 54-62, 2002.

[10] J. Gómez-Gardeñes, S. Gómez, A. Arenas, and Y. Moreno, "Explosive synchronization transitions in scale-free networks," Physical Review Letters, vol. 106, no. 12, Article ID 128701, 2011.

[11] T. K. D. M. Peron and F. A. Rodrigues, "Determination of the critical coupling of explosive synchronization transitions in scale-free networks by mean-field approximations," Physical Review E-Statistical, Nonlinear, and Soft Matter Physics, vol. 86, no. 5, Article ID 056108, 5 pages, 2012.

[12] J. Cao and L. Li, "Cluster synchronization in an array of hybrid coupled neural networks with delay," Neural Networks, vol. 22, no. 4, pp. 335-342, 2009.

[13] A. Arenas, A. Díaz-Guilera, J. Kurths, Y. Moreno, and C. Zhou, "Synchronization in complex networks," Physics Reports, vol. 469, no. 3, pp. 93-153, 2008.

[14] J. B. Zhang, Z. J. Ma, and G. Zhang, "Cluster synchronization induced by one-node clusters in networks with asymmetric negative couplings," Chaos, vol. 23, no. 4, Article ID 043128, 7 pages, 2013.

[15] K. Z. Li, M. Small, and X. C. Fu, "Generation of clusters in complex dynamical networks via pinning control," Journal of Physics A: Mathematical and Theoretical, vol. 41, no. 50, Article ID 505101, 17 pages, 2008.

[16] L. L. Li and J. D. Cao, "Cluster synchronization in an array of coupled stochastic delayed neural networks via pinning control," Neurocomputing, vol. 74, no. 5, pp. 846-856, 2011.
[17] W. Wu and T. P. Chen, "Partial synchronization in linearly and symmetrically coupled ordinary differential systems," Physica D: Nonlinear Phenomena, vol. 238, no. 4, pp. 355-364, 2009.

[18] J. B. Zhang, Z. J. Ma, and J. D. Cao, "Full synchronization studied by a set of partitions connected together," Abstract and Applied Analysis, vol. 2013, Article ID 352826, 8 pages, 2013.

[19] Z. J. Ma, Z. R. Liu, and G. Zhang, "A new method to realize cluster synchronization in connected chaotic networks," Chaos, vol. 16, no. 2, Article ID 023103, 9 pages, 2006.

[20] Y. Wang and J. Cao, "Cluster synchronization in nonlinearly coupled delayed networks of non-identical dynamic systems," Nonlinear Analysis: Real World Applications, vol. 14, no. 1, pp. 842-851, 2013.

[21] J. B. Zhang, Z. J. Ma, and G. Zhang, "Control schemes for synchronizing two subnetworks with weak couplings," Chinese Physics B, vol. 23, no. 1, Article ID 010507, 7 pages, 2014.

[22] X. Yang, J. Cao, and Z. Yang, "Synchronization of coupled reaction-diffusion neural networks with time-varying delays via pinning-impulsive controller," SIAM Journal on Control and Optimization, vol. 51, no. 5, pp. 3486-3510, 2013.

[23] J. Cao, D. W. C. Ho, and Y. Yang, "Projective synchronization of a class of delayed chaotic systems via impulsive control," Physics Letters A, vol. 373, no. 35, pp. 3128-3133, 2009.

[24] J. Lu, J. Kurths, J. Cao, N. Mahdavi, and C. Huang, "Synchronization control for nonlinear stochastic dynamical networks: pinning impulsive strategy," IEEE Transactions on Neural Networks and Learning Systems, vol. 23, no. 2, pp. 285-292, 2012.

[25] J. J. Wu, Z. U. Gao, and H. J. Sun, "Cascade and breakdown in scale-free networks with community structure," Physical Review E-Statistical, Nonlinear, and Soft Matter Physics, vol. 74, no. 6, Article ID 066111, 6 pages, 2006.

[26] H. J. Sun, H. Zhang, and J. J. Wu, "Correlated scale-free network with community: modeling and transportation dynamics," Nonlinear Dynamics, vol. 69, no. 4, pp. 2097-2104, 2012.

[27] W. L. Lu and T. P. Chen, "New approach to synchronization analysis of linearly coupled ordinary differential systems," Physica D: Nonlinear Phenomena, vol. 213, no. 2, pp. 214-230, 2006. 


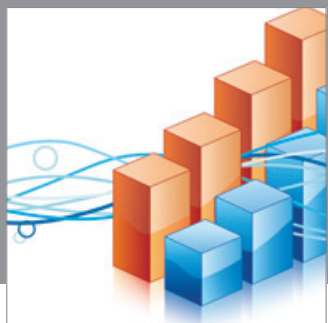

Advances in

Operations Research

mansans

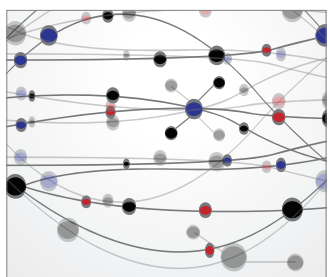

The Scientific World Journal
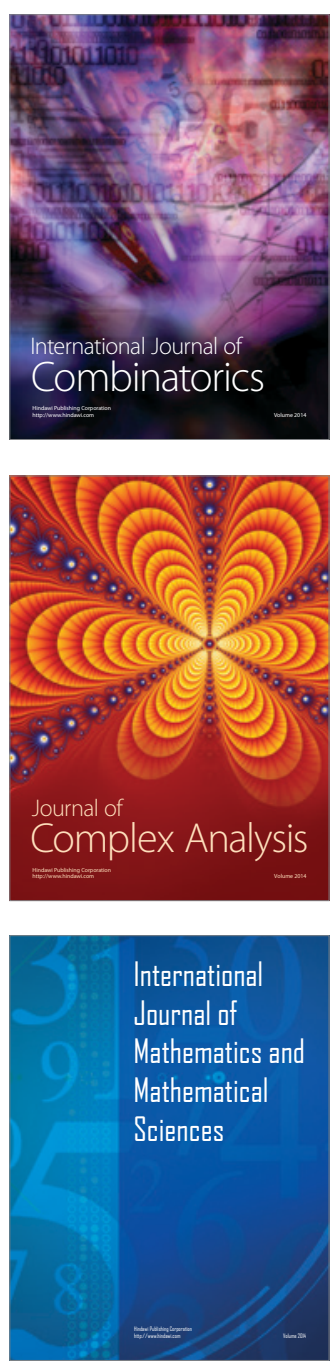
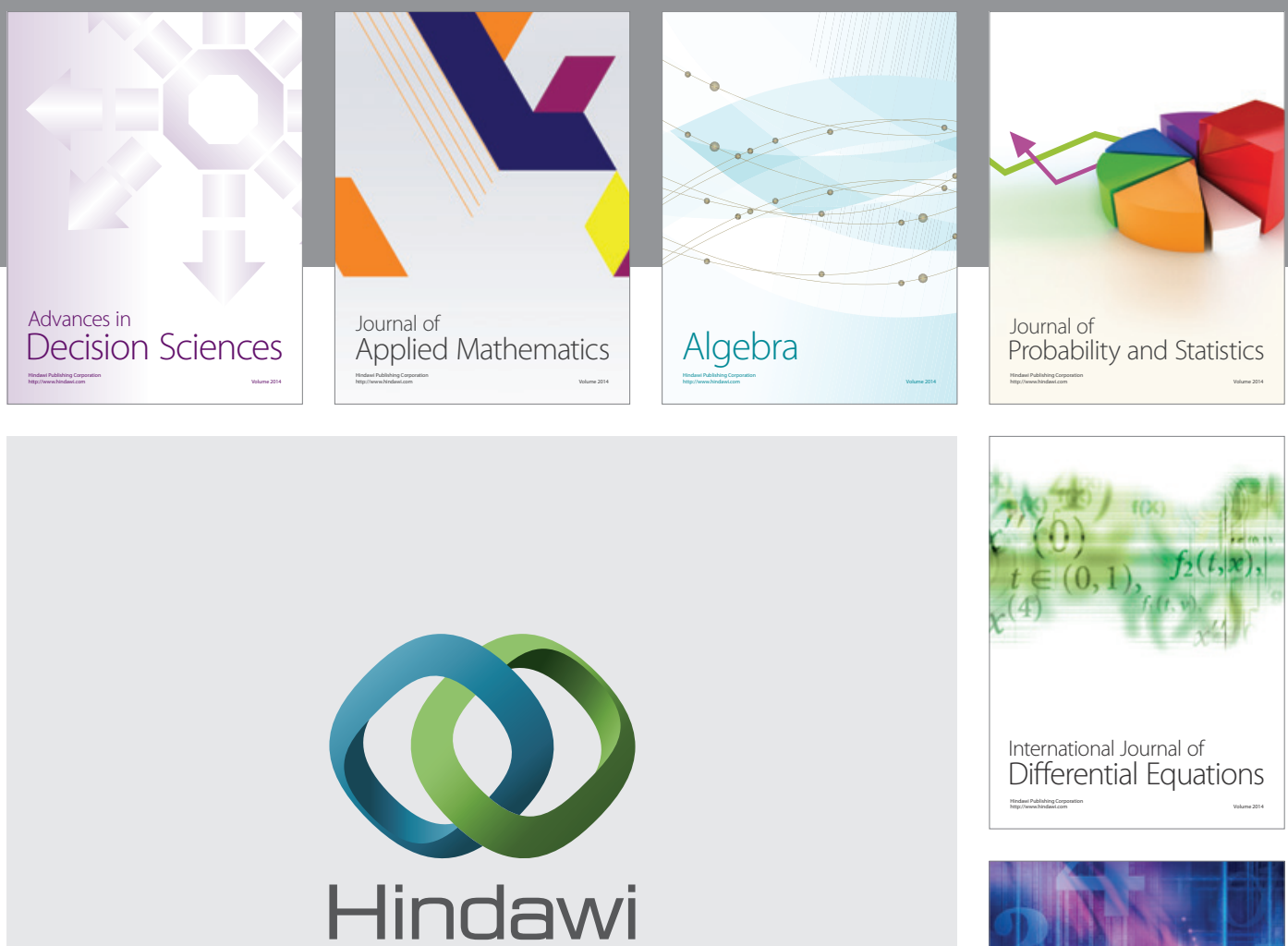

Submit your manuscripts at http://www.hindawi.com
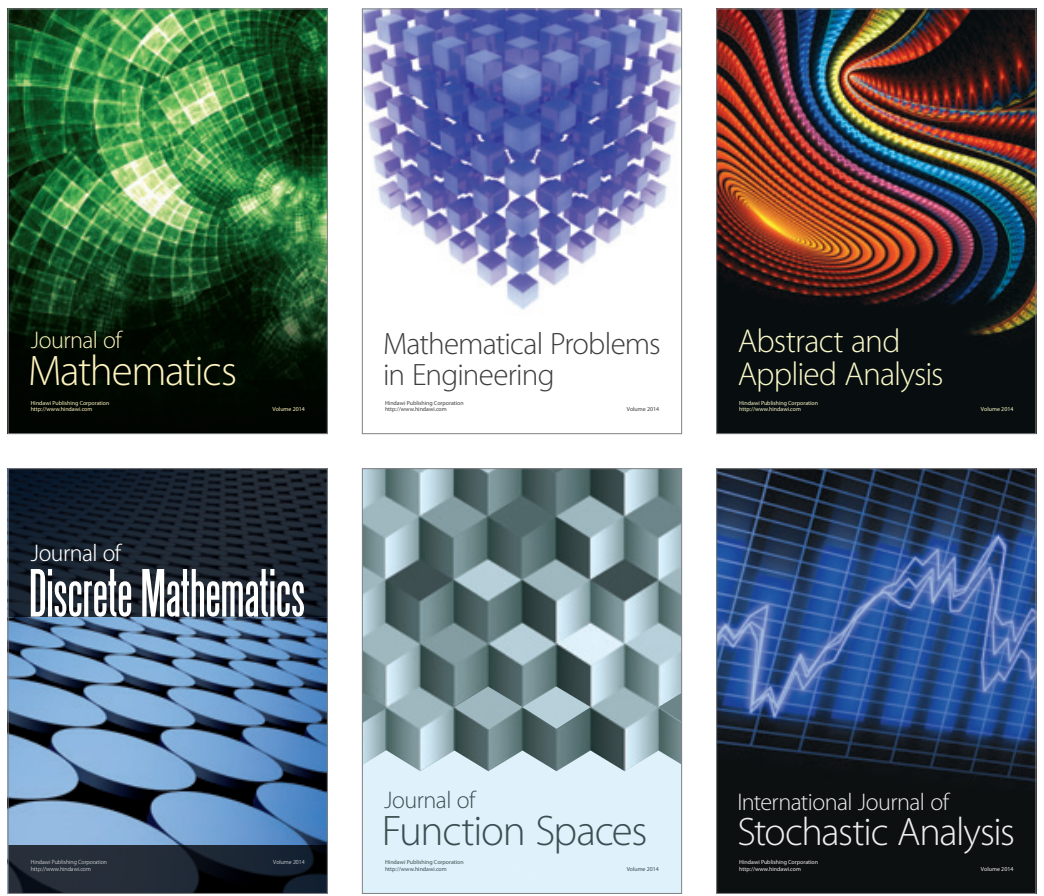

Journal of

Function Spaces

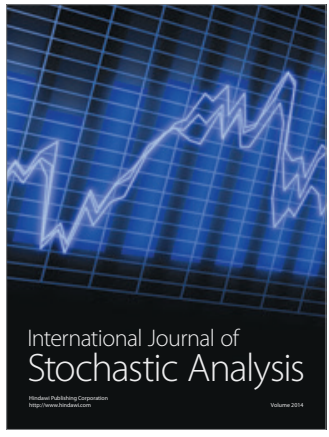

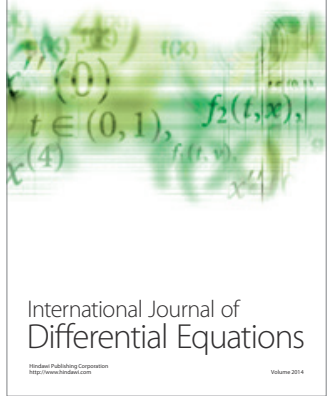
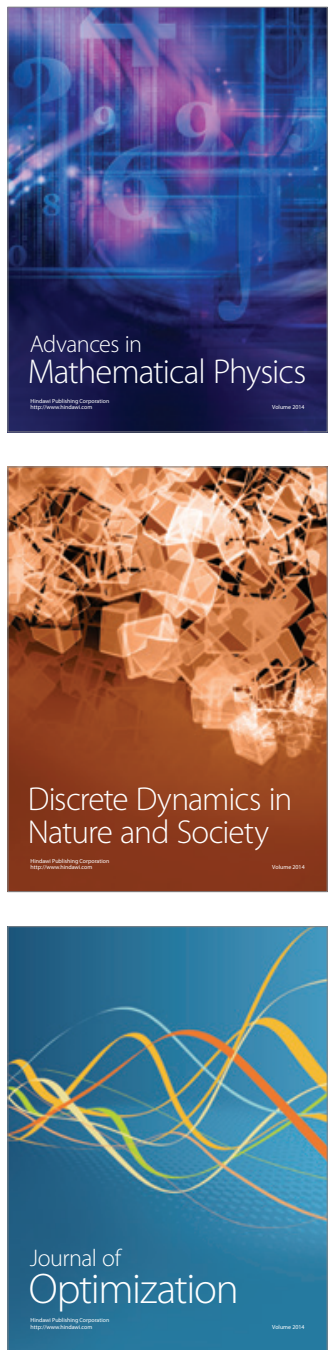